\title{
Acidic Leucine-Rich Nuclear Phosphoprotein 32 Family Member A
}

National Cancer Institute

\section{Source}

National Cancer Institute. Acidic Leucine-Rich Nuclear Phosphoprotein 32 Family Member

A. NCl Thesaurus. Code C125587.

Acidic leucine-rich nuclear phosphoprotein 32 family member A (249 aa, 29 kDa) is encoded by the human ANP32A gene. This protein plays a role in enzyme inhibition, transcriptional regulation, apoptosis, mRNA localization and stability, cell proliferation and differentiation, and tumor suppression. 